
LA-UR- 93
Title:
Cyclodextrin-Based Microsensors for Volatile organic
Compounds CONF-970443-

Author(s):

Basil Swanson, Sabina Johnson, Jeane Shi, and Xiaoguang Yang

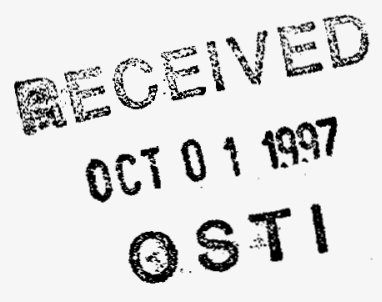

Submitted to:

Publication in ACS Symposium Series
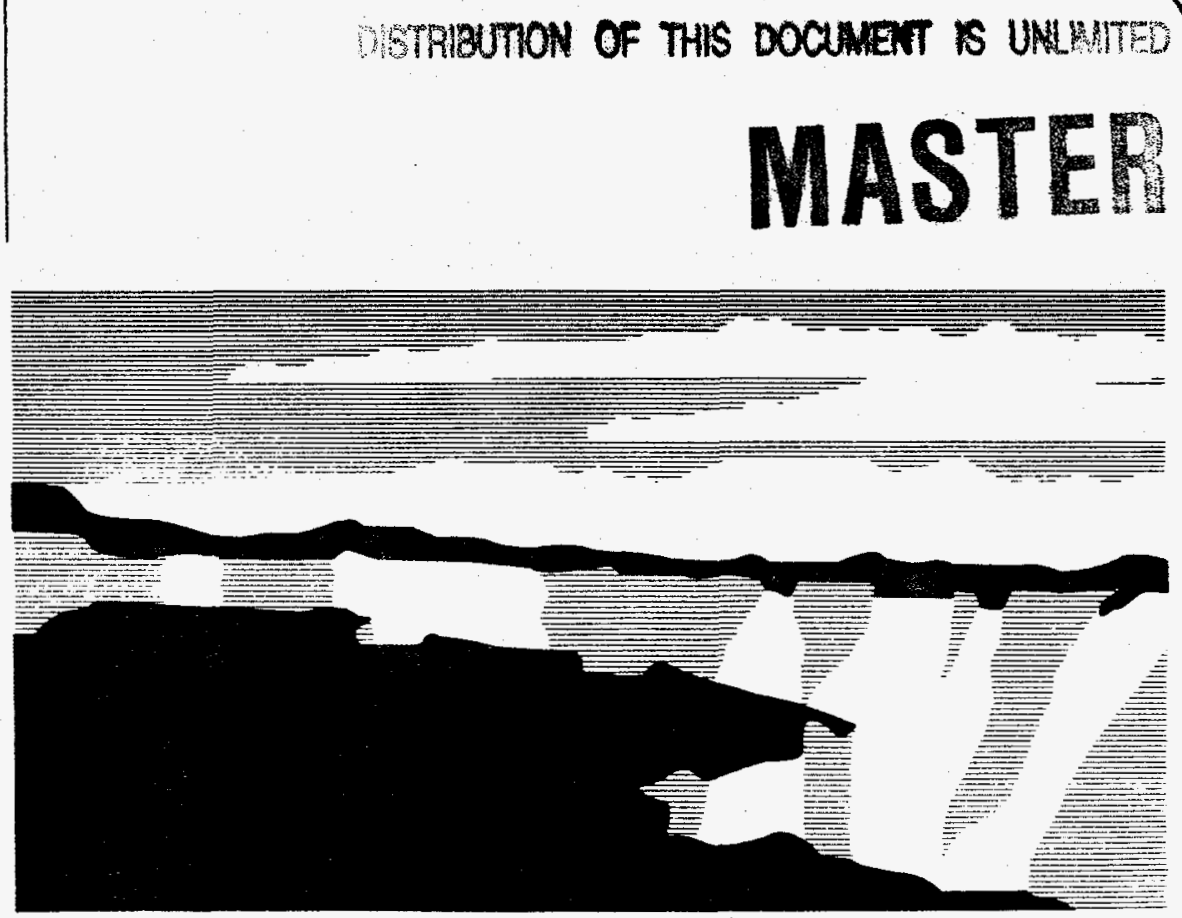


\section{DISCLAIMER}

This report was prepared as an account of work sponsored by an agency of the United States Government. Neither the United States Government nor any agency thereof, nor any of their employees, make any warranty, express or implied, or assumes any legal liability or responsibility for the accuracy, completeness, or usefulness of any information, apparatus, product, or process disclosed, or represents that its use would not infringe privately owned rights. Reference herein to any specific commercial product, process, or service by trade name, trademark, manufacturer, or otherwise does not necessarily constitute or imply its endorsement, recommendation, or favoring by the United States Government or any agency thereof. The views and opinions of authors expressed herein do not necessarily state or reflect those of the United States Government or any agency thereof. 


\section{DISCLAMIER}

Portions of this document may be illegible in electronic image products. Images are produced from the best available original document. 


\title{
Cyclodextrin-Based Microsensors for Volatile Organic Compounds
}

\author{
B. Swanson", S. Johnson, J. Shi, X. Yang \\ Chemical Science and Technology, Los Alamos National Laboratory \\ Los Alamos, NM 87545, USA
}

\begin{abstract}
Host-guest chemistry and self-assembly techniques are being explored to develop species selective thin-films for real-time sensing of volatile organic compounds (VOCs). Cyclodextrin (CD) and calixarene (CA) molecules are known to form guest-host inclusion complexes with a variety of organic molecules. Through the control of the cavity size and chemical functionality on the rims of these bucket-like molecules, the binding affinities for formation of inclusion complexes can be controlled and optimized for specific agents. Self-assembly techniques are used to covalently bond these reagent molecules to the surface of acoustic transducers to create dense, highly oriented, and stable thin films. Self-assembly techniques have also been used to fabricate multilayer thin film containing molecular recognition reagents through alternating adsorption of charged species in aqueous solutions. Self-assembly of polymeric molecules on the SAW device was also explored for fabricating species selective interfaces.
\end{abstract}

The quest for microchemical and biosensors has focused the need to create highly selective and highly sensitive interfaces. Various chemical species such as metals (1), metal oxides (2), metal complexes (3), organic host molecules (4), and functionalizd polymers (5) have been deposited on the transducer surface and studied as molecular recognition reagents for sensing chemical species in gas phase. Among them, organic host molecules containing a hydrophobic cavity attract increasing attention in sensor development for real-time monitoring of volatile organic compounds (VOCs). These organic receptors bind VOCs through noncovalent interactions such as van der Waals interactions and hydrogen bonding, relying heavily on their hydrophobic cavity

In our research on VOC sensor development, we aim to achieve highly selective and sensitive sensors based on host-guest chemistry. By changing the functionality and the cavity sizes of CDs, chemical specificity of host-guest interactions can be achieved. Three approaches were studied to incorporate cyclodextrin (CD) molecular recognition reagents into interfaces on surface acoustic wave (SAW) device (6). The first relies on molecular self-assembly monolayer technique to couple CDs covalently to the transducer surface as densely packed and highly orientated monolayers. The 
second approach employs polyelectrolyte self-assembly technique to fabricate organized multilayer interfaces containing CDs and CAs. The third approach explores in-situ polymerization reactions to cross-link derivatized $\mathrm{CD}$ molecules onto the transducer surface.

Molecular recognition reagents. Cyclodextrins (CDs) are semi-natural products and are produced on the industrial scales. CDs have a preorganized rigid cavity with three different sizes and the hydroxyl groups on the rims of the cavity allow for various chemical transformations (Figure 1). CDs are best known to form host-guest complexes in solutions, especially in aqueous media, with organic species (7). However, derivatized cyclodextrins immobilized on capillary columns have been used for separations of a variety of organic species in gas phases at elevated temperatures $\left(>50^{\circ} \mathrm{C}\right)(8)$. The fact that organic enantiomers can be resolved by $\mathrm{CD}$ coated columns confirms that $\mathrm{CDs}$ bind the gas species in their cavities (8a) A recent theoretical calculation on the permethylated beta-CD revealed that the host-guest interaction is mainly controlled by van der Waals forces (9). In the gas phase the host-guest electronic complementarity, steric complementarity (shape recognition) and the host preorganization are three key elements in determining the stability constant of a supramolecular complex (10).

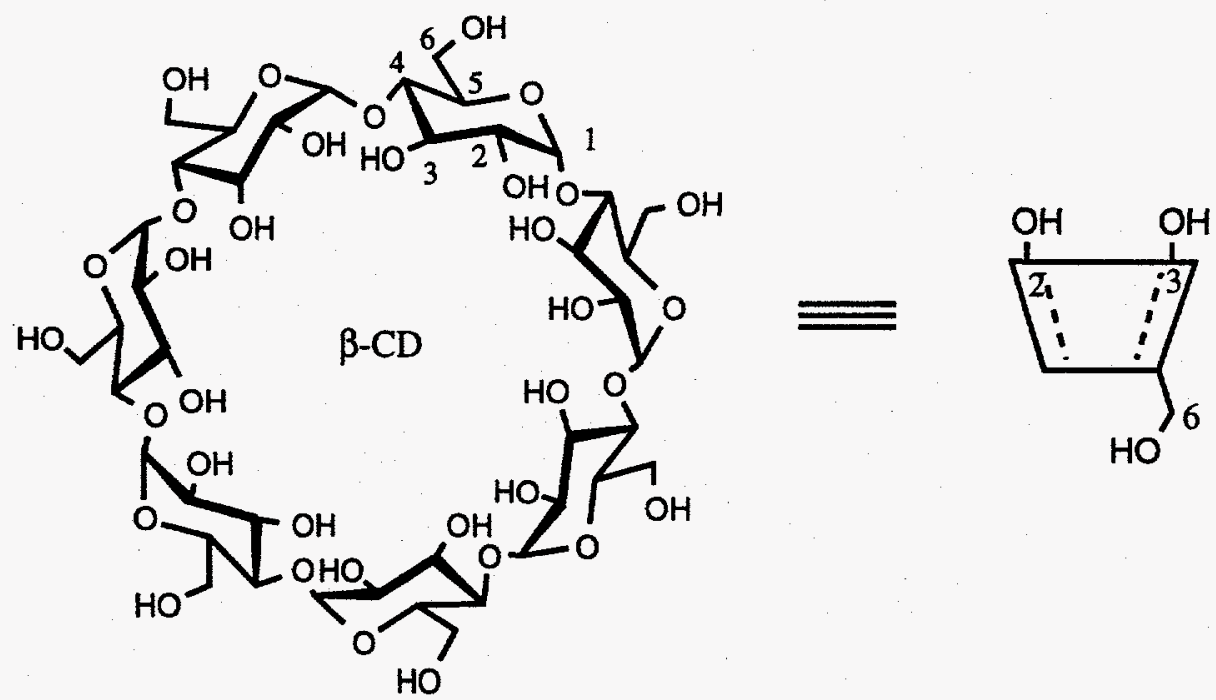

Figure 1. Molecular structure of $\beta$-cyclodextrin and schematic representation of the cyclodextrin shape and secondary hydroxyl groups on upper rims and primary hydroxyl groups on the lower rim.

Functionalized cyclodextrins have been studied for various applications such as enzyme mimics, separations, and drug delivery (11). The binding characteristics of CDs change upon functionalization of the hydroxyl groups, because of changes in the 
shape, size and charge distributions of the cavity and loss of certain intramolecular hydrogen bonding (12). By creating a series of alkylated cyclodextrins and leaving the primary hydroxyl groups unchanged for future coupling to the transducer surface, we were able to study the different binding affinities towards VOCs based on the changes in the R group (Figure 2) and utilize these differences to create chemically specific sensors.
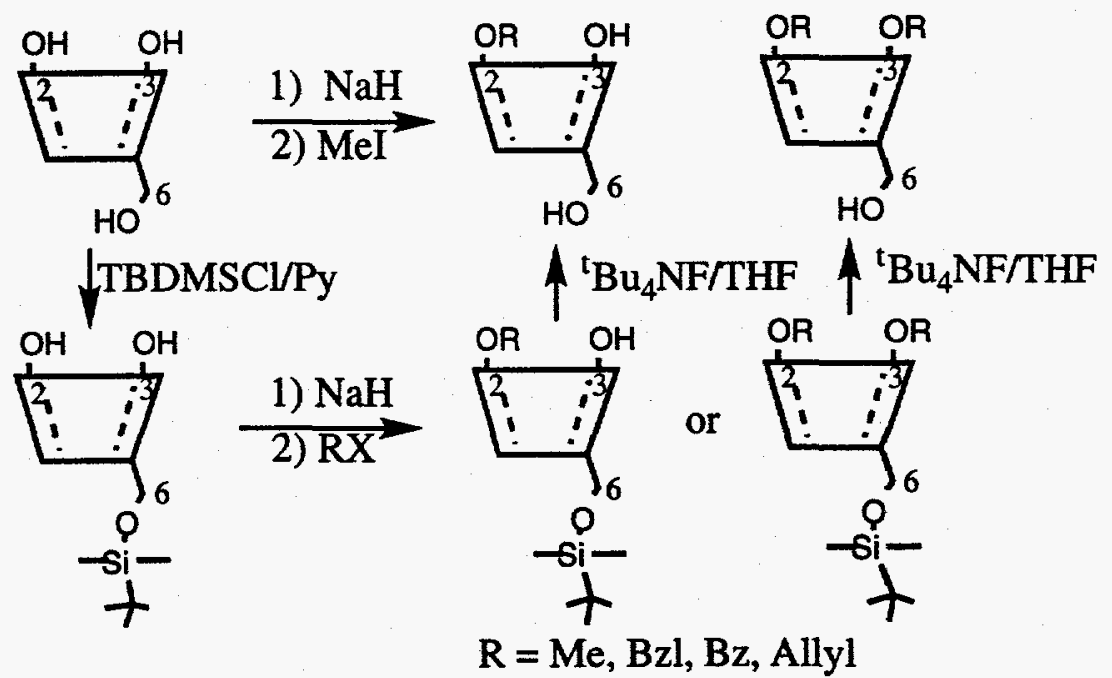

Figure 2. Chemical transformations of cyclodextrins (13).

Self-assembled monolayers as sensing interfaces. SAMs or LB films have been used as a linker layer to immobilize enzymes and antibodies on biosensors (14). Similarly, we explored the possibility to use SAMs as linker layers to covalently couple cyclodextrin reagents to the transducer surface, in this case, surface acoustic wave device. It has been recognized that silanes with long alkyl chains form SAMs with organized crystalline structures. A bromo-terminated SAM was formed by the immersion of a quartz substrate into a solution of 11-bromoundecyltrichlorosilane or 16-bromo-hexadecyltrichlorosilane hexadecane solution. The nucleophilic substitution reaction with potassium cyanate gave a isocycanate-terminated surface, which reacts in situ with primary hydroxyl groups on the CDs (Figure 3). The monolayers were characterized by ellipsometry measurement, contact angle measurement and FT-IR.

Several SAW devices coated with monolayer cyclodextrins were tested for sensing volatile organic compounds. Figure 4 is the real time responses of a heptakis(2-O-benzyl)- $\beta$-cyclodextrin SAW device to halogenated hydrocarbons. The sensor response is reversible, rapid and linear, as shown in the case of perchloroethylene (Figure 4(D) and 4(E)). The sensor is much more sensitive towards perchloroethylene and toluene (not shown here) and less sensitive to chloroform and 1,1,1-trichloroethane which are relatively larger in size. We also found that the sensitivity of the device is much lower towards smaller molecules such as methanol, 

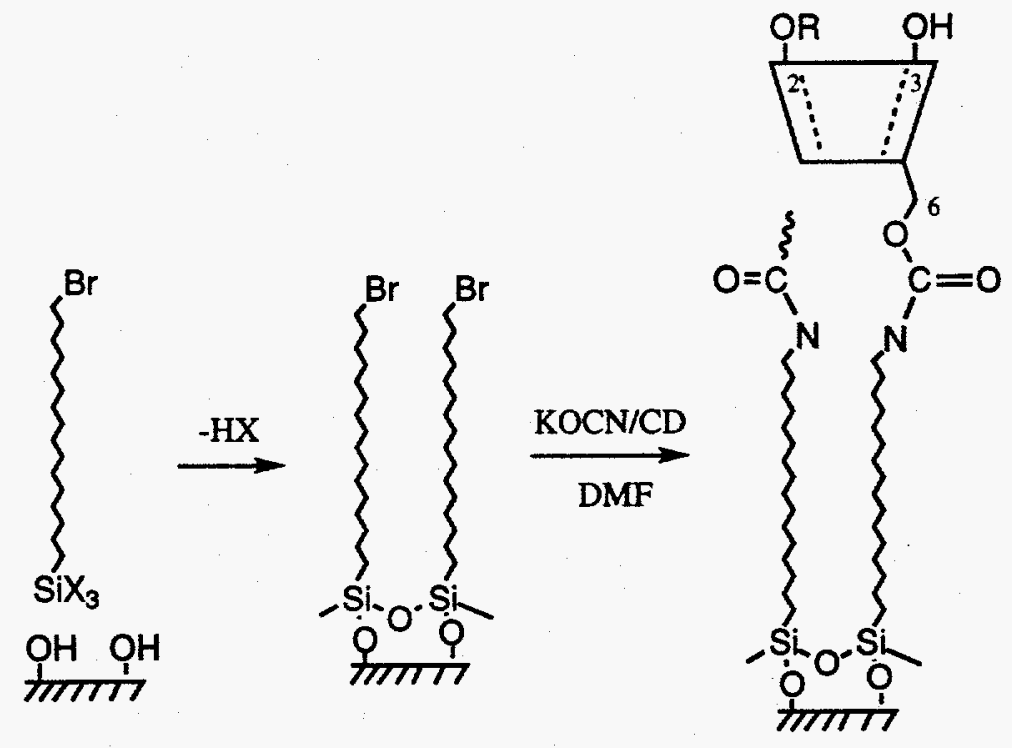

Figure 3. Formation of bromo-terminated SAM and coupling of cyclodextrins to the transducer surface.

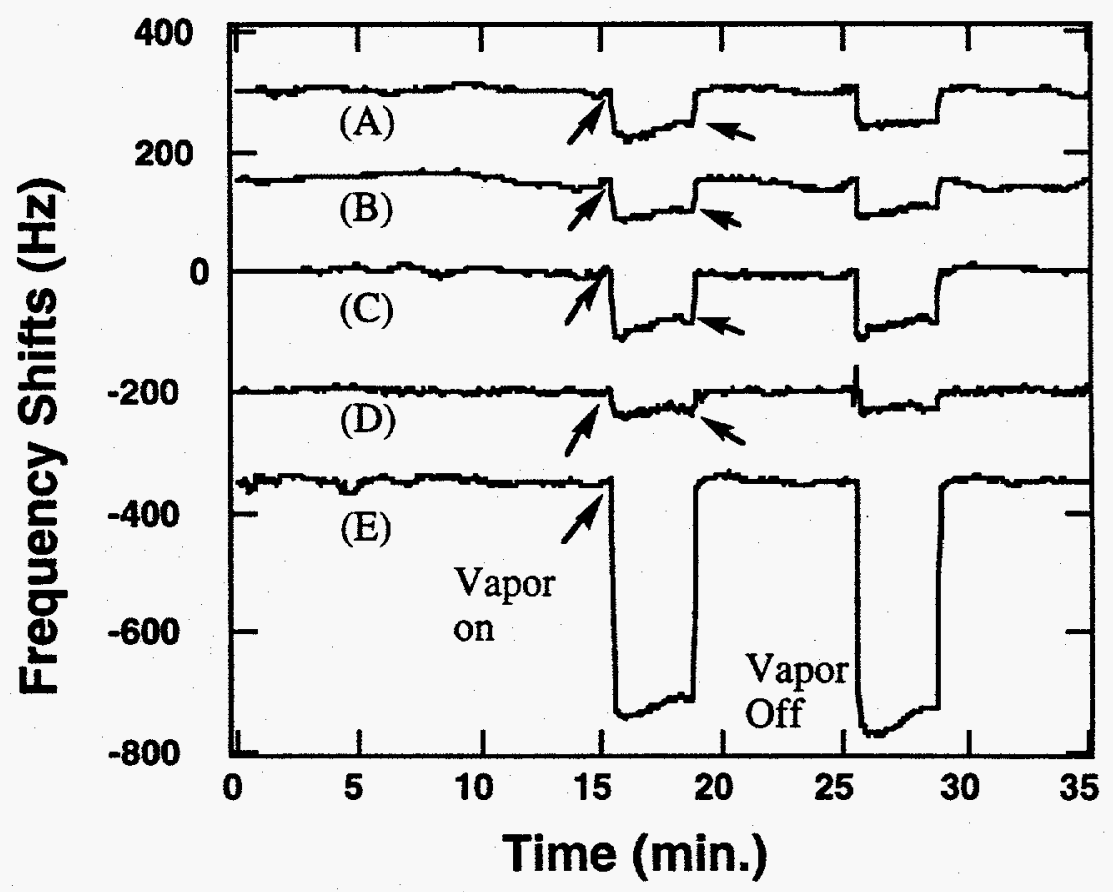

Figure 4. Real time SAW responses to halogenated hydrocarbons: (A) $65 \mathrm{~Hz}$ at $1115 \mathrm{ppm} \mathrm{1,1,1-trichloroethane;} \mathrm{(B)} 58 \mathrm{~Hz}$ at $552 \mathrm{ppm}$ trichloroethylene; (C) $103 \mathrm{~Hz}$ at $1644 \mathrm{ppm}$ chloroform; (D) $38 \mathrm{~Hz}$ at $138 \mathrm{ppm}$ perchloroethylene; (E) $377 \mathrm{~Hz}$ at $1381 \mathrm{ppm}$ perchloroethylene. The device is coated with heptakis(2$O$-benzyl- $\beta$-cyclodextrin). 
hexane and acetone. In general, monolayer $\mathrm{CD}$ coated devices can detect VOC vapors at about $100 \mathrm{ppm}$ concentration.

\section{Polyelectrolyte deposition approach to mutilayer CD and CA films. For} developing selective sensor coatings containing molecular recognition reagents such as cyclodextrins, calixarenes, cavitands, metalloporphyrins, there exist problems in fabricating ordered multilayer molecular films. Solution casting of molecular species results in films with low coverage, poor uniformity, low stability and low reproducibility. We studied a recently developed technique using polyelectrolyte selfassembly to grow organic mutilayer films. This technique consists of alternating adsorption of anionic and cationic electrolytes on a charge substrate by sequential dipping of the substrate into aqueous polyelectrolyte solutions. The films formed are uniform, organized, durable and reproducible in terms of film thickness (15).

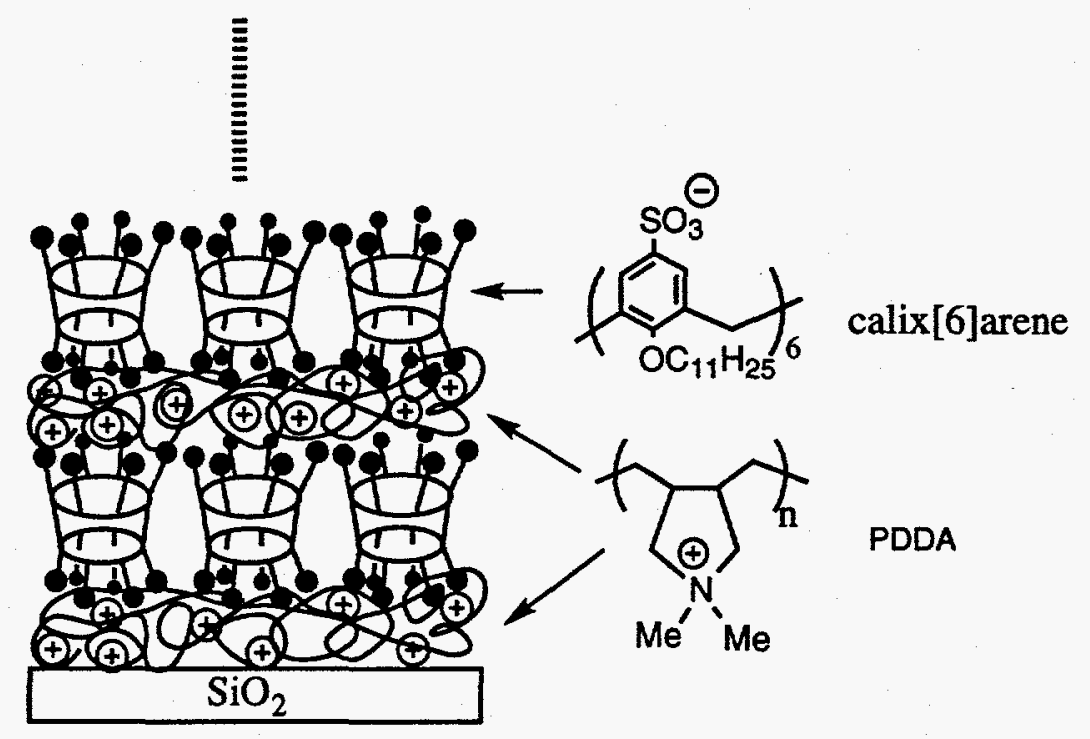

Figure 5. Alternating polyelectrolyte PDDA and p-sulfonated calix[6]arene depositions on a surface-modified $\mathrm{SiO}_{2}$ surface.

Multilayer thin films with precisely controlled thickness and molecular architectures can therefore be successfully fabricated. Replacing polyanions, highly negatively charged molecular recognition reagents were employed for film fabrication. This unique approach incorporates polymer and molecular elements into the sensing film and thus results in films with polymer's physical properties and molecular film's selectivity. Multilayer molecular films of polyelectrolyte/calixarene and polyelectrolyte/cyclodextrin hosts were fabricated by alternating adsorption of charged species in aqueous solutions onto a substrate (quartz or silicon wafer). $250 \mathrm{MHz}$ SAW device was first treated with aminopropyltrimethoxysilane, followed with deposition of poly(sodium 4-styrenesulfonate) (PSS) and then poly(dimethyldiallyl-ammonium) (PDDA). After this, alternating depositions of negatively charged molecular host species (Figure 5) and 
PDDA were carried out until the desired number of bilayers was reached. Between each deposition, the device was thoroughly rinsed with deionized water. For film characterizations, silicon wafers or quartz substrates were used for film depositions. This layer-by-layer molecular deposition approach has been successfully employed to integrate molecular recognition reagents into the polymer films as chemically selective layers. The films were characterized with SEM, infrared and mass loading were monitored by SAW devices. These measurements revealed that the deposition process is highly reproducible and the resulting films are uniform and stable.

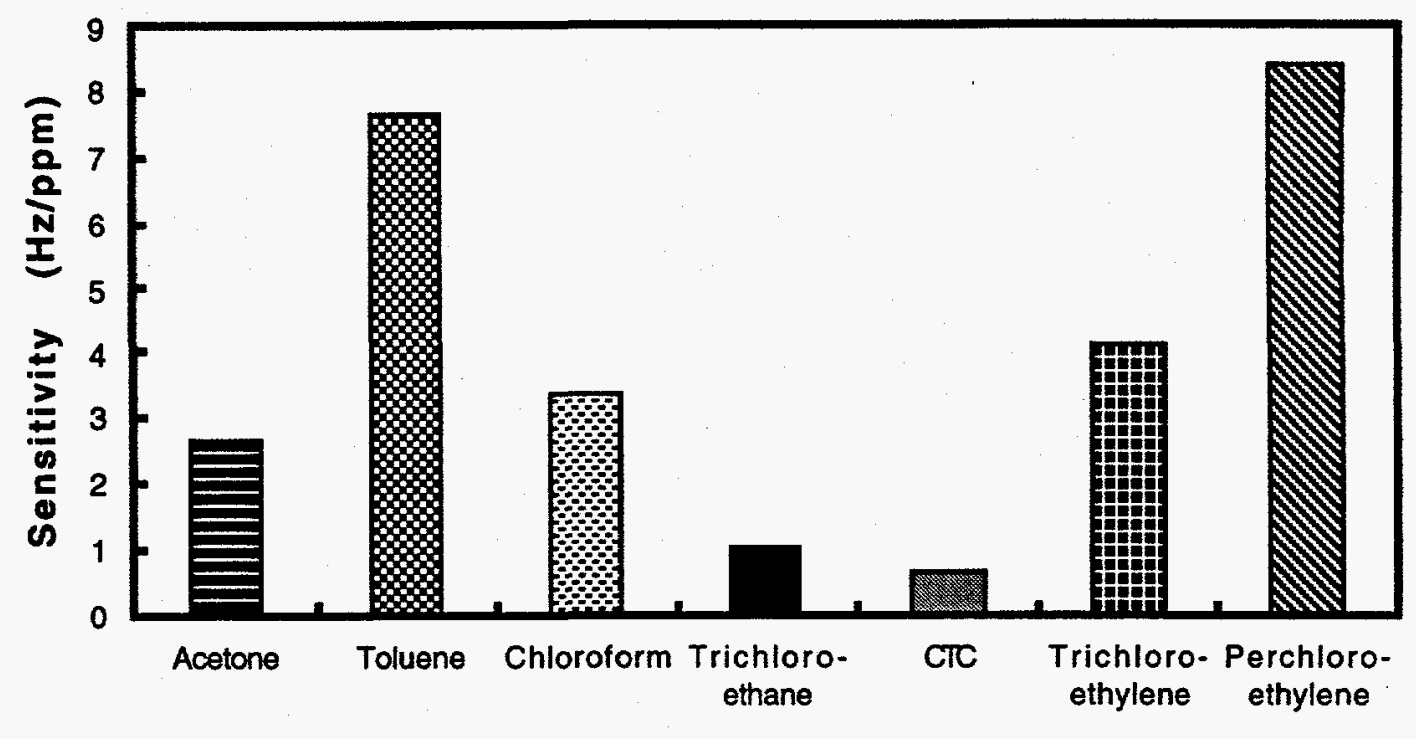

\section{Volatile Organic Compounds}

Figure 6. Sensor sensitivity to volatile organic compounds. The sensor was coated with five bilayer of $\mathrm{p}$-sulphonated calix[6]arene/PDDA.

SAW sensors coated with calix[6]arene sulfonate/PDDA (Figure 5) or sulphated $\beta$-cyclodextrin/PDDA were studied for sensing a variety of organic solvent vapors. It was found that sensor coated with the calixarene showed increased sensitivity with the increasing number of calixarene layers deposited on the SAW device. However, the sensitivity to carbon tetrachloride and 1,1,1-trichloroethane increases only slightly. The sensor responses to perchloroethylene (PCE) increase with increasing number of the calix[6]arene layers. The result indicates that, in addition to the surface layer, the vapor sorption also involves inner layer species. The five bilayer calix[6]arene film exhibts high sensitivity towards PCE, toluene and trichloroethylene (Figure 6). The sensor can detect vapors at sub-ppm concentrations. On the other hands, a SAW device coated with polyelectrolytes only (PDDA/PSS) didn't show increased sensitivity upon increasing thickness of the film and the sensitivity is much lower ( 3 to 4 times less). There is no selectivity observed with this film. We also fabricated a sulphated $\beta$-cyclodextrin bilayer film with PDDA. The sensor was not particularly sensitive towards hydrophobic organic vapors, which 
might result from high charge density and hydrophilic nature on the $C D$ rim. Other charged $\mathrm{CD}$ species are now being pursued.

In-situ formation of polysiloxane cyclodextrin films on the surface. This is an in-situ polymerization approach to multilayer selective and sensitive sensing layers. The purpose of using a siloxane formation approach is two-fold. First, this provides a simple and easily reproducible approach to multilayer films for optimization of sensitivity. Secondly, the use of a siloxane intervoid network to minimize adsorption of target VOCs into interstitial sites that will act as competitive binding sites relative to the cyclodextrin cavities. The hydrophobic VOCs would prefer to be adsorbed in the molecular host cavities. In general, SAW devices were coated by immersing the devices in a DMF solution of trimethoxysilane functionized CDs overnight (Figure 7). The devices was then cured in the air at $60^{\circ} \mathrm{C}$ for $30 \mathrm{~min}$. The mass loading of the polymers on SAW devices ranges from 128 to $138 \mathrm{kHz}$, which corresponding to the film thickness of 11 to $13 \mathrm{~nm}$. The films are uniform and amorphous, as revealed by SEM.
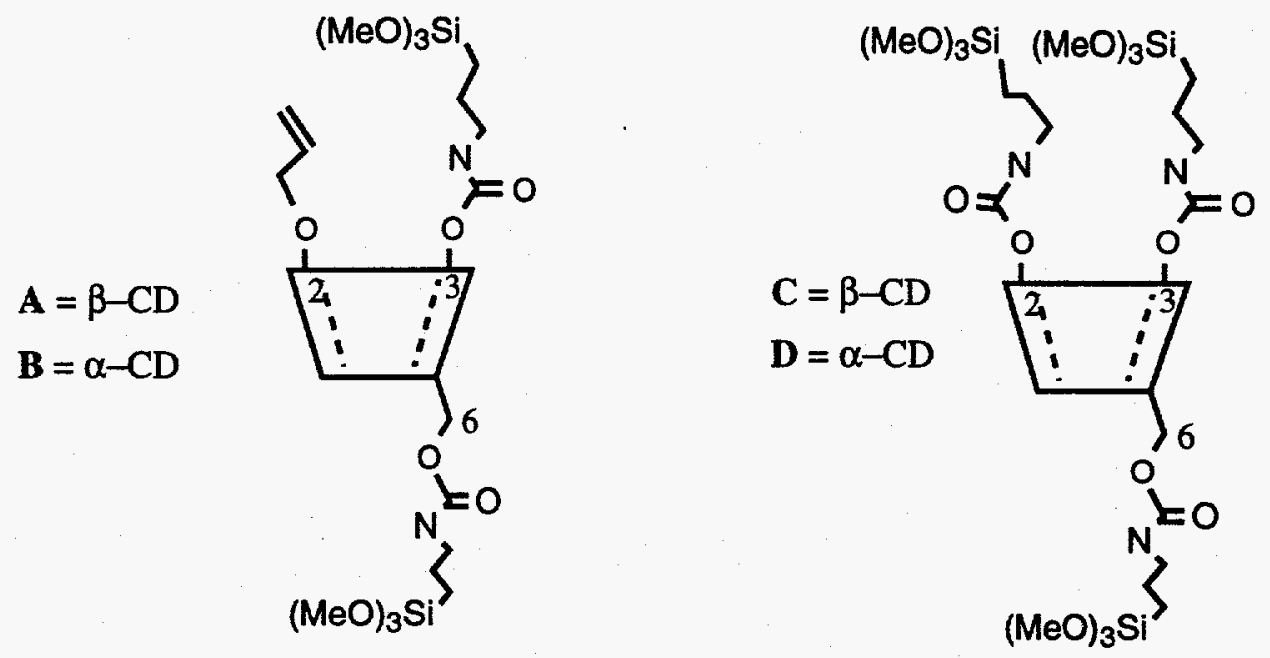

Figure 7. Siloxane $\mathrm{CD}$ polymer precursors.

The siloxane $\mathrm{CD}$ coated devices are sensitive and selective toward several VOCs. The response time is in seconds of the exposure to the analytes and the responses are reversible. $\mathrm{CD}$-siloxane coated SAW sensors can detect analytes (perchroloethylene, trichloroethylene, trichloroethane, etc.) in tens of ppm without difficulty. We also tested the stability of these $\mathrm{CD}$-siloxane coated devices. After 2 months, no significant sensitivity loss was observed. Figure 8 shows response patterns for eight vapors over four CD-siloxane coated SAW sensors. These eight 


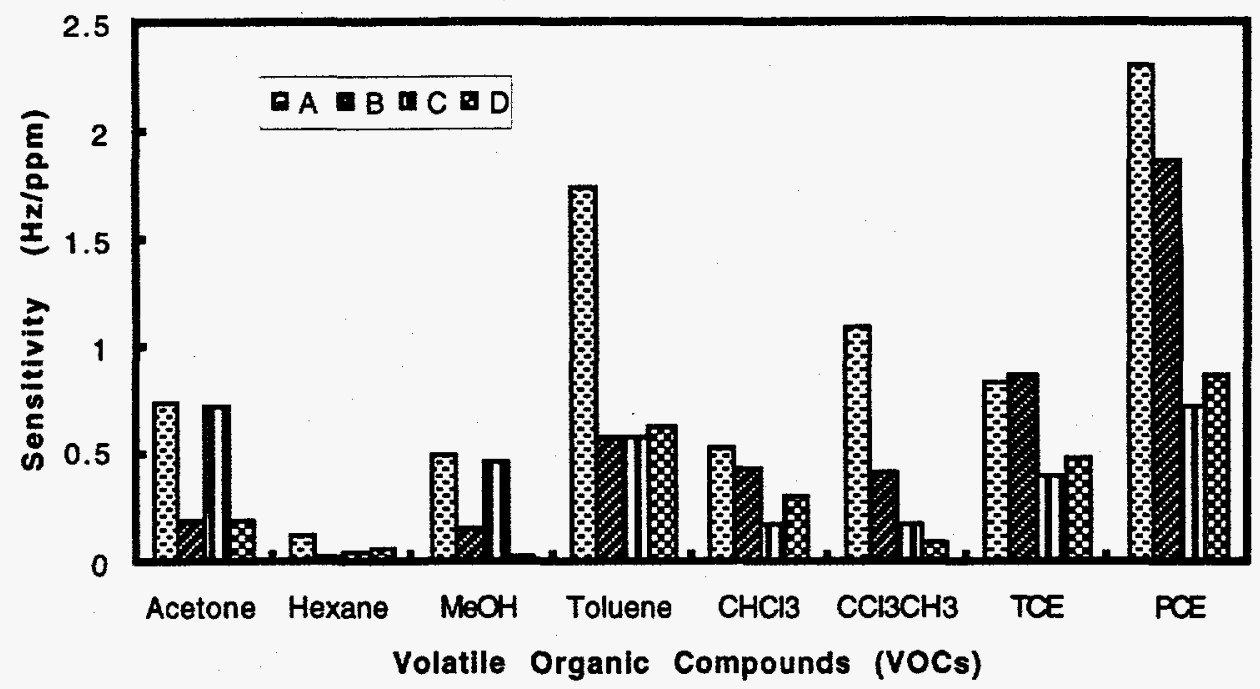

Figure 8. Sensor array response patterns to VOCs with four $250 \mathrm{MHz} \mathrm{SAW}$ devices coated with siloxane $C D$ polymer precursors $A, B, C$, and $D$, as shown in Figure 7.

vapors can be grouped into three, according to their response patterns. As expected, they are grouped by their chemical structures: nonpolar hydrocarbons (hexane and toluene), polar hydrocarbons (acetone and methanol), and halogenated hydrocarbons (chloroform, trichloroethane, and perchloroethylene and TCE). Hexane has a very low sensitivity, compared with toluene. Four halogenated hydrocarbons, though similar, have their distinct patterns. $\alpha-C D$ coated SAW sensor is more selective to toluene and hexane than $\beta-C D$ coated sensor and on the other hand, $\beta-C D$ coated sensor is more selective to halogenated hydrocarbon than $\alpha-C D$. The resulted selectivity due to $C D$ cavity size difference indicates that vapors do recognize their hosts at the gas-solid interface. The affinity of toluene toward $\alpha-C D$ and PCE towards $\beta-C D$ is well documented in the solution studies and solid state structures.

\section{Conclusion}

Three approaches have been studied to fabricate chemically selective interfaces for sensing volatile organic compounds. By incorporating molecular recognition reagents, cyclodextrins and their derivatives, into sensing layers, SAW sensors with high sensitivity and selectivity can be obtained. Further work includes tailoring of cyclodextrins and construction of organized sensing mutilayers and minimizing nonspecific binding. 


\section{Acknowledgments}

This work was supported by Laboratory Directed Research and Development (LDRD) funds and the Advanced Technologies for Proliferation Detection program of the Nuclear Nonproliferation Office of the Department of Energy.

\section{References}

1. D'Amico, A.; Palma, A.; Vetelino, E. Sens. Actuators 1982, 3, 31.

2. Bryant, A.; Poirier, M.; Riley, G.; Lee, D. L.; Vetelino, J. F. Sens. Actuators 1983, $4,105$.

3. (a) Zeller, E. T.; Zhang, G. Anal. Chem. 1992, 64, 1277; (b) Nieuwenhuizen, M. S.; Harteveld, J. L. Talanta 1994, 41, 461.

4. (a) Lai, C. S.; Moody, G. J. Thomas, J. D. R J. Chem. Soc. Perkin Trans. 1988, 319; (b) Bodenhöfer, K.; Hierlemann, A.; Noetzel, G.; Weimar, U.; Göpel, W. Anal. Chem. 1996, 68, 2210; (c) Schierbaum, K. D.; Weiss, T.; Thoden, E. U.; Velzen, T.; Engbersen, J. F. J.; Reinhoudt, D. N.; Göpel, W. Science 1994, 265, 1413; (d) Moore, L. W.; Springer, K.; Shi, J.; Yang, X.; Swanson, B. I.; Li, D. Adv. Mater. 1995, 7, 729;

5. McGill, R. A.; Abraham, M. H.; Grate, J. W. Chemtech 1994, 27.

6. Wohltjen, H.; Dessy, R. E. Anal. Chem. 1979, 51, 1458;

7. Saenger, W. in Inclusion Compounds; Atwood, J. L.; Davis, J. E. D; MacNicol, D. D., Ed.; Academic Press, London, 1984, Vol.2; pp 231-260.

8. (a) König, W. A.; Lutz, S.; Hagen, M.; Krebber, R.; Wenz, G.; Baldenius, K.; Ehlers, J.; Dieck, H. tom. J. High Res. Chromatorg. 1989, 12, 35; (b) Schurig, V.; Schmalzing, D.; Schleimer, M. Angew. Chem. Ed. Int. Engl. 1991, 30, 987. 13. Vincenti, M.; Dalcanale, E.; Sonicini, P.; Guglielmentti, G. J. Am. Chem. Soc. 1990, $112,445$.

9. Lipkowitz, K. B.; Pearl, G.; Coner, B.; Peterson, M. A. J. Am. Chem. Soc. 1997, 119,600 .

10. Diederich, F. Cyclophanes The Royal Society of Chemistry, London, 1991.

11. Szejtli, J. in Inclusion Compounds; Atwood, J. L.; Davis, J. E. D,; MacNicol, D. D., Ed.; Academic Press, London, 1984, Vol. 3; pp 331-391.

12. Harata, K. in Inclusion Compounds; Atwood, J. L.; Davis, J. E. D,; MacNicol, D. D., Ed.; Academic Press, London, 1991, Vol.5; pp 311-344.

13. (a) Takeo, K.; Mitoh, H.; Uemura, K. Carbohydrate Research 1989, 187, 203; (b) Rong, D.; D'Souza, V. T. Tetrahedron Lett. 1990, 31, 4273.

14. Thompson, M.; Krull, U. J. Anal. Chem. 1991, 63, 393A.

15. (a) Decher, G.; Hong, J. D. Ber. Bunsenges. Phys. Chem. 1991, 95, 1430; (b) Decher, G.; Hong, J. D.; Schmitt, J. Thin Solid Films 1992, 210/211, 831; (c) Lvov, Y.; Decher, G.; Möhwald, H. Langmuir 1993, 9, 481. 